\title{
QCD Equation of State and Dark Matter
}

\section{Mark Hindmarsh ${ }^{* 1}$ and Owe Philipsen ${ }^{2}$}

${ }^{1}$ Dept. of Physics \& Astronomy,

University of Sussex,

Brighton BN1 9QH, UK

${ }^{2}$ Institut für Theoretische Physik,

Westfälische Wilhelms-Universität Münster,

48149 Münster, Germany

Email: m.b.hindmarsh@sussex.ac.uk, ophil@uni-muenster.de

The QCD equation of state is not often discussed in cosmology. However, the relic density of weakly interacting massive particles (WIMPs) depends on the entropy and the expansion rate of the Universe when they freeze out, at a temperature in the range $400 \mathrm{MeV}-40 \mathrm{GeV}$, where QCD corrections are still important. We use recent analytic and lattice calculations of the QCD pressure to produce a new equation of state suitable for use in relic density calculations. As an example, we show that relic densities calculated by the dark matter package DarkSUSY receive corrections of several per cent, within the observational accuracy of the Planck CMB mission, due for launch in 2007 .

29th Johns Hopkins Workshop on current problems in particle theory: Strong matter in the heavens, Budapest, Hungary

August, 1-3 2005

${ }^{*}$ Speaker. 


\section{Introduction}

In the early Universe, Weakly Interacting Massive Particles (WIMPs, see e.g. [1]) of mass $m$ generically freeze out of equilibrium at a temperature of around $T_{f} \simeq m / 25 \mathrm{GeV}$. For masses in the range $10-1000 \mathrm{GeV}, T_{f}$ lies between $400 \mathrm{MeV}$ and $40 \mathrm{GeV}$. The relic density depends on both energy and entropy densities near $T_{f}$, and so the WIMP relic density is sensitive to the equation of state of the Universe in this temperature range.

We re-examine the equation of state for the Universe in this critical regime using recent work in both the deconfined [2] and confined [3] phases, and investigate the consequences for the WIMP relic density in one of the dark matter packages, DarkSUSY [4]. Replacing the standard-issue equation of state (used in both DarkSUSY and MicrOMEGAs [5]) which is based on Refs. [6, 7, 8], with ours gives corrections to the relic densities calculated in the supplied test code of several percent. WMAP has already determined the matter density to better than $10 \%$ in single-field inflation models [9] and Planck promises to do much better, with one estimate at less than 1\% [10], although other authors find that Sloan Digital Sky Survey (SDSS) data is required for this level of accuracy [11].

\section{Equation of state of QCD}

Work on the pressure of non-Abelian gauge theories has a long history $[12,13,14,15,16]$. Perturbative expansions in the coupling constant $g$ of quantities such as the pressure $p$ converge badly, and particularly for a strongly-coupled theory like QCD. Strictly perturbative expansions, even when expanded to $\mathrm{O}\left(g^{6} \ln g\right)$ [2], seem to work only at very high temperatures, above about $10^{5} T_{c}$ (where $T_{c}$ is the critical temperature).

However, by constructing a sequence of effective theories for the scales $2 \pi T, g T$ and $g^{2} T$, one can match the calculated pressure reasonably well to pure-glue lattice data near the critical temperature by fitting for a non-perturbative and as yet unknown $\mathrm{O}\left(g^{6}\right)$ term [2]. Below the phase transition the hadronic resonance gas model, which models the plasma as an ideal gas of mesons, baryons and their excited states, matches reasonably well to lattice data [3].

Near the transition, lattice calculations exist for the pressure and energy density for $N_{f}=0,2$, $2+1$, and 3 , although only the pure glue case has been extrapolated to the continuum. The critical temperatures (defined as the peak of the susceptibility) are currently given as $T_{c}(0)=271 \pm 2 \mathrm{MeV}$, $T_{c}(2)=173 \pm 8 \mathrm{MeV}$ and $T_{c}(3)=154 \pm 8 \mathrm{MeV}[17]$

\section{Relic density calculations}

Consider a particle of mass $m$ and number density $n$, undergoing annihilations $X X \rightarrow \ldots$ with total cross-section $\sigma$ at temperature $T$. Then [18]

$$
\dot{n}+3 H n=-\left\langle\sigma v_{\mathrm{M} \varnothing \mathrm{l}}\right\rangle\left(n^{2}-n_{\mathrm{eq}}^{2}\right),
$$

where $n_{\mathrm{eq}}$ is the equilibrium number density, $H=\dot{a} / a$ is the Hubble parameter, and $v_{\mathrm{M} \varnothing \mathrm{l}}$ is the Møller velocity. For two particles with velocities $\mathbf{v}_{1}$ and $\mathbf{v}_{2}$,

$$
v_{\mathrm{M} \varnothing \mathrm{l}}=\left(\left(\mathbf{v}_{1}-\mathbf{v}_{2}\right)^{2}-\left(\mathbf{v}_{1} \times \mathbf{v}_{2}\right)^{2}\right)^{\frac{1}{2}},
$$


It is convenient to convert the time variable to $x=m / T$, and to measure the relic abundance in terms of $Y=n / s$, where $s$ is the entropy density. If the total entropy $S=s a^{3}$ is conserved, then we can write [18]

$$
\frac{d Y}{d x}=\frac{1}{3 H} \frac{d s}{d x}\left\langle\sigma v_{\mathrm{M} \varnothing \mathrm{l}}\right\rangle\left(Y^{2}-Y_{\mathrm{eq}}^{2}\right)
$$

where $Y_{\mathrm{eq}}$ is the equilibrium value of $Y, H=\dot{a} / a$ is the Hubble parameter, $a$ is the scalar factor of the expanding background. The adiabaticity assumption is violated if the QCD transition is first order, but it is most likely to be a cross-over transition at the low chemical potentials which are relevant for the early Universe $[14,15]$.

Using the Friedmann equation $H^{2}=8 \pi G \rho / 3$, and defining effective numbers of degrees of freedom for the energy and entropy densities through

$$
\rho=\frac{\pi^{2}}{30} g_{\mathrm{eff}}(T) T^{4}, \quad s=\frac{2 \pi^{2}}{45} h_{\mathrm{eff}}(T) T^{3},
$$

one finds that the relic density depends on the parameter

$$
g_{*}^{1 / 2}(T)=\frac{h_{\mathrm{eff}}}{g_{\mathrm{eff}}^{1 / 2}}\left(1+\frac{T}{3} \frac{d \ln h_{\mathrm{eff}}}{d T}\right)
$$

\section{Effective degrees of freedom and their effect on relic densities}

If the Standard Model at high temperature behaved as an ideal gas, the computation of $g_{\text {eff }}$ and $h_{\text {eff }}$ proceeds straightforwardly. The contributions from a particle of mass $m_{i}$ to the energy density $\rho$ and the entropy density $s=(\rho+p) / T$ are given by

$$
\begin{aligned}
g_{i, \mathrm{eff}} & \equiv \frac{\rho_{i}}{\rho_{0}}=\frac{15}{\pi^{4}} \int_{x_{i}}^{\infty} \frac{\left(u^{2}-x_{i}^{2}\right)^{\frac{1}{2}}}{e^{u} \pm 1} u^{2} d u, \\
h_{i, \mathrm{eff}} & \equiv \frac{s_{i}}{s_{0}}=\frac{45}{12 \pi^{4}} \int_{x_{i}}^{\infty} \frac{\left(u^{2}-x_{i}^{2}\right)^{\frac{1}{2}}}{e^{u} \pm 1}\left(4 u^{2}-x_{i}^{2}\right) d u,
\end{aligned}
$$

where $\rho_{0}$ and $s_{0}$ are the ideal energy and entropy densities for a free boson. These contributions are summed. In practice there would still be some uncertainty, as the masses of the quarks are not accurately known (with the exception of the top quark).

Interactions in non-Abelian plasmas correct the ideal, with the dominant corrections coming from the coloured degrees of freedom. Their contribution to the pressure is scaled by a function $f(T)$, defined to be the ratio between the true QCD pressure $p(T)$ and the Stefan-Boltzmann result $p_{\mathrm{SB}}$ for the same theory, $f(T)=p(T) / p_{\mathrm{SB}}(T)$. This correction factor is derived from lattice [19] and perturbative [2] calculations for $N_{f}=0$, and uses an approximate universality in the pressure curves for different $N_{f}$ observed by Karsch et al. [19]:

$$
p / p_{0}=f_{\mathrm{QCD}}\left(T / T_{c}\left(N_{f}\right)\right),
$$

where $T_{c}\left(N_{f}\right)$ is the critical temperature for the theory with $N_{f}$ light fermion flavours. Besides this, there appears to be only negligible $N_{f}$-dependence within the current numerical accuracy. We are therefore motivated to neglect quark mass effects, take the $N_{f}=0$ lattice data (in the continuum 

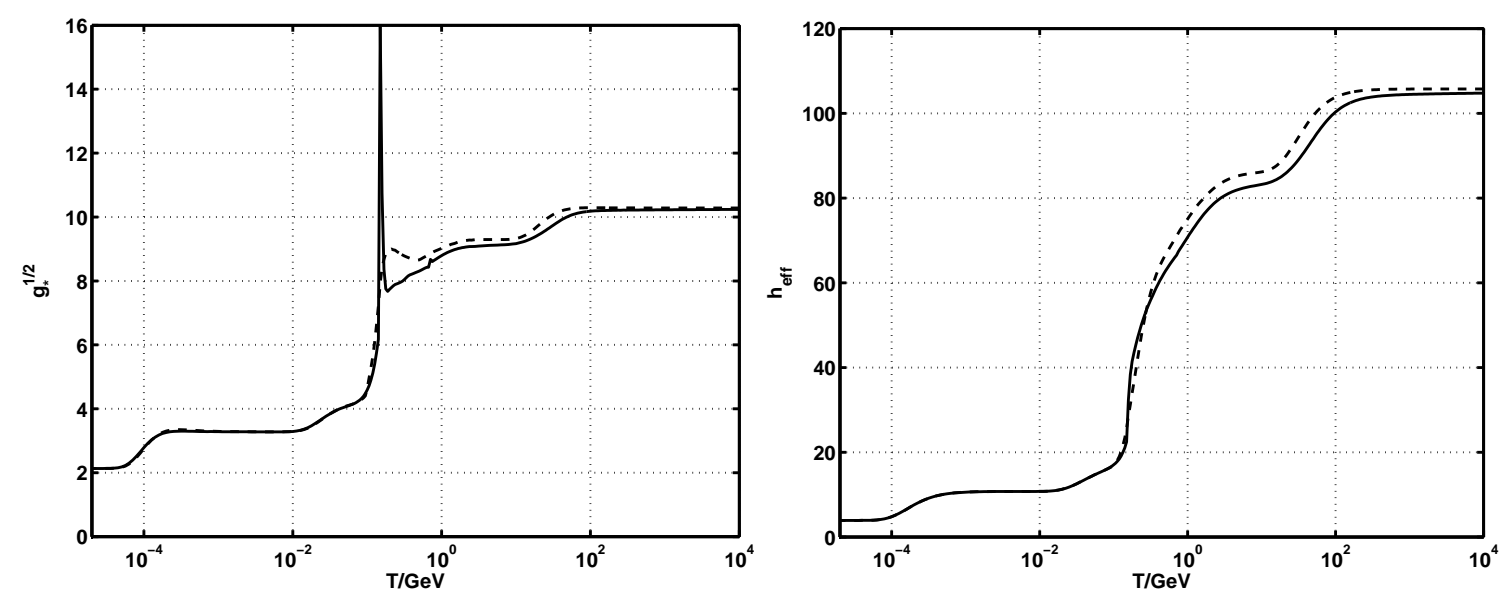

Figure 4.1: Degrees of freedom factors $g_{*}^{1 / 2}(T)$ and $h_{\text {eff }}(T)$, defined in the text, for our equation of state B (solid) compared with those currently used in DarkSUSY and MicrOMEGAs (dashed). The spike in the upper panel is a numerical artefact, reflecting uncertainty in the true equation of state.

limit) and the $N_{f}=0 \mathrm{DR}$ formula of Kajantie et al. [2], and scale the temperature dependence by $T_{c}(3) / T_{c}(0)$. The correction factors are matched at $1.2 \mathrm{GeV}$ using the undetermined $\mathrm{O}\left(g^{6}\right)$ parameter, which we take to be 0.6755 , close to the value 0.7 used in Ref. [2].

In the confined phase we label our model equations of state (EOS) A,B and C. EOS A ignores hadrons completely, as the lattice shows that $f(T)$ very rapidly approaches zero below $T_{c}$. EOS $\mathrm{B}$ and $\mathrm{C}$ model hadrons as a gas of free mesons and baryons. It was noted in Ref. [3] that such a gas, including all resonances, gives a pressure which fits remarkably well to the $N_{f}=2+1$ lattice results although, as the authors themselves point out, this result should be treated with caution as the simulations are not at the continuum limit. We include all resonances listed in the Particle Data Group's table mass_width_02.mc [20].

We make a sharp switch to the hadronic gas at a temperature $T_{\mathrm{HG}}$. For our EOS B we take $T_{\mathrm{HG}}=T_{c}=154 \mathrm{MeV}$, and for EOS C we take $T_{\mathrm{HG}}=200 \mathrm{MeV}$ and $T_{c}=185.5 \mathrm{MeV}$, values chosen to give as smooth a curve for $h_{\text {eff }}$ as possible. The effects of these equations of state on the relic densities turn out to differ by less than $0.3 \%$ in the relevant temperature interval, so in the following we concentrate on EOS B.

In Figs. 1 we plot for EOS B our calculated effective numbers of degrees of freedom $h_{\mathrm{eff}}(T)$ and $g_{*}^{1 / 2}(T)$ defined in Eqs. 3.4 and 3.5, compared with those used in DarkSUSY [4] and MicrOMEGAs [5], leading packages for calculating the properties of supersymmetric dark matter. We see that there are differences, particularly near the QCD transition, which is perhaps not surprising as the QCD equation of state used in DarkSUSY [8] is quite old [6, 7]. The spike in $g_{*}^{1 / 2}(T)$ is due to the sharp transition between the high and low temperature equations of state and is probably exaggerated.

In Table 4.1 we exhibit the effect of the new equation of state on the density of relic neutralinos $\chi$, for the mSUGRA models used to test DarkSUSY in the standard distribution [21]. We find changes of about $1.5-3.5 \%$. In order to quantify the effect of uncertainty in the lattice data, we introduce two new models B2 and B3, which are constructed by scaling the lattice curve by 0.9 


\begin{tabular}{l|c|c|c|c|c} 
Model & $m_{\chi} / \mathrm{GeV}$ & $T_{f} / \mathrm{GeV}$ & $\Omega_{c} h^{2}(\mathrm{DS})$ & $\Omega_{c} h^{2}(\mathrm{here})$ & $\Delta(\%)$ \\
\hline $\mathrm{A}^{\prime}$ & 242.83 & 9.8 & 0.0929 & $0.0948_{(42)}^{(54)}$ & $2.0_{1.4}^{2.6}$ \\
$\mathrm{~B}^{\prime}$ & 94.88 & 4.1 & 0.1213 & $0.1242_{(36)}^{(56)}$ & $2.4_{1.5}^{3.6}$ \\
$\mathrm{C}^{\prime}$ & 158.09 & 6.5 & 0.1149 & $0.1174_{(63)}^{(83)}$ & $2.2_{1.5}^{2.9}$ \\
$\mathrm{D}^{\prime}$ & 212.42 & 8.6 & 0.0864 & $0.0882_{(76)}^{(88)}$ & $2.0_{1.4}^{2.7}$ \\
$\mathrm{G}^{\prime}$ & 147.98 & 6.2 & 0.1294 & $0.1323_{(13)}^{(33)}$ & $2.2_{1.4}^{3.0}$ \\
$\mathrm{H}^{\prime}$ & 388.38 & 16.0 & 0.1629 & $0.1662_{(51)}^{(71)}$ & $2.0_{1.5}^{2.6}$ \\
$\mathrm{I}^{\prime}$ & 138.08 & 5.8 & 0.1319 & $0.1351_{(40)}^{(62)}$ & $2.4_{1.6}^{3.2}$ \\
$\mathrm{~J}^{\prime}$ & 309.17 & 12.6 & 0.0966 & $0.0984_{(79)}^{(90)}$ & $2.0_{1.4}^{2.5}$ \\
$\mathrm{~K}^{\prime}$ & 554.19 & 22.9 & 0.0863 & $0.0883_{(78)}^{(88)}$ & $2.3_{1.8}^{3.0}$ \\
$\mathrm{~L}^{\prime}$ & 180.99 & 7.5 & 0.0988 & $0.1011_{(03)}^{(18)}$ & $2.3_{1.5}^{3.0}$ \\
\hline
\end{tabular}

Table 4.1: Relic densities in benchmark models [23], calculated with DarkSUSY. Displayed: neutralino mass $m_{\chi}$, freeze-out temperature $T_{f}, \Omega_{c} h^{2}$ computed using the current DarkSUSY equation of state, $\Omega_{c} h^{2}$ using our QCD-corrected Equations of State $\mathrm{B}_{(\mathrm{B} 3)}^{(\mathrm{B} 2)}$, and percentage changes $\Delta$.

and 1.1 respectively, and then adjusting the $\mathrm{O}\left(g^{6}\right)$ parameter in the DR pressure curve so that it meets the scaled lattice curve at $T=4.43 T_{c}$. Thus a $10 \%$ uncertainty in the lattice pressure curve translates to an uncertainty in the relic density in the range $0.5-1 \%$. Note that the lowest freezeout temperature in the table is about $4 \mathrm{GeV}$. This corresponds to more than $20 T_{c}$, where the QCD corrections to $g_{*}$ are around 5\%. Evidently, WIMPs with freeze-out temperatures closer to $T_{c}$ would be affected more strongly. The production of sterile neutrinos is also affected by the details of the QCD transition [22].

\section{Conclusions}

By updating the equation of state of the Standard Model in the light of recent developments in high temperature QCD, we have found differences in WIMP relic density calculations of a few per cent for selected models in the DarkSUSY. Although the effect is small, it is not insignificant. In single field inflation models, the 68\% confidence limit on the matter density is already $\Omega h^{2}=$ $0.127 \pm 0.017$ from the WMAP First Year data [9], while Planck has been estimated to be able to reach a level of $\Delta \Omega h^{2}=0.0011$ in the most optimistic of circumstances [10].

Given a sufficiently accurate $g *$, we speculate that with a combination of measurements of the neutralino mass and cross-sections in direct detection experiments and at LHC and the ILC, the effective number of degrees of freedom in the early Universe at a few $\mathrm{GeV}$ will become experimentally accessible, motivating attempts to improve further on this work.

\section{Acknowledgments}

We are indebted to F. Karsch and J. Engels for lattice data, to M. Laine for providing details from Ref. [2], and to P. Gondolo and F. Boudjema for discussions. We acknowledge support from 
PPARC and from the European Network for Theoretical Astroparticle Physics (ENTApP), member of ILIAS, EC contract number RII-CT-2004-506222 (MH).

\section{References}

[1] G. Bertone, D. Hooper and J. Silk, Particle dark matter: Evidence, candidates and constraints, Phys. Rept. 405 (2005) 279, [hep-ph / 0404175$].$

[2] K. Kajantie, M. Laine, K. Rummukainen and Y. Schroder, The pressure of hot QCD up to $g * * 6$ $\ln (1 / g)$, Phys. Rev. D67 (2003) 105008, [hep-ph/0211321].

[3] F. Karsch, K. Redlich and A. Tawfik, Hadron resonance mass spectrum and lattice QCD thermodynamics, Eur. Phys. J. C29 (2003) 549, [hep-ph/0303108].

[4] P. Gondolo et al., DarkSUSY: Computing supersymmetric dark matter properties numerically, (2004), [astro-ph/0406204].

[5] G. Belanger, F. Boudjema, A. Pukhov and A. Semenov, MicrOMEGAs: Version 1.3, (2004), [hep-ph/ 0405253$]$.

[6] K. A. Olive, The thermodynamics of the Quark-Hadron phase transition in the early Universe, Nucl. Phys. B190 (1981) 483.

[7] K. A. Olive, D. N. Schramm and G. Steigman, Limits on new superweakly interacting particles from primordial nucleosynthesis, Nucl. Phys. B180 (1981) 497.

[8] M. Srednicki, R. Watkins and K. A. Olive, Calculations of relic densities in the early universe, Nucl. Phys. B310 (1988) 693.

[9] H. V. Peiris et al., First year Wilkinson Microwave Anisotropy Probe (WMAP) observations: Implications for inflation, Astrophys. J. Suppl. 148 (2003) 213, [astro-ph/0302225].

[10] A. Balbi, C. Baccigalupi, F. Perrotta, S. Matarrese and N. Vittorio, Probing dark energy with the CMB: projected constraints from MAP and Planck, Astrophys. J. 588 (2003) L5, [astro-ph/0301192].

[11] J. Lesgourgues, S. Pastor and L. Perotto, Probing neutrino masses with future galaxy redshift surveys, (2004), [hep-ph/0403296].

[12] J.-P. Blaizot, E. Iancu and A. Rebhan, Thermodynamics of the high-temperature quark gluon plasma, (2003), [hep-ph/0303185].

[13] U. Kraemmer and A. Rebhan, Advances in perturbative thermal field theory, Rept. Prog. Phys. 67 (2004) 351, [hep-ph/0310337].

[14] F. Karsch, Lattice QCD at high temperature and density, Lect. Notes Phys. 583 (2002) 209, [hep-lat/0106019].

[15] E. Laermann and O. Philipsen, Status of lattice QCD at finite temperature, Ann. Rev. Nucl. Part. Sci. 53 (2003) 163, [hep-ph/ 0303042$].$

[16] J. O. Andersen and M. Strickland, Resummation in hot field theories, (2004), [hep-ph/0 404164 ].

[17] F. Karsch, E. Laermann and A. Peikert, Quark mass and flavor dependence of the QCD phase transition, Nucl. Phys. B605 (2001) 579, [hep-lat/ 0012023 ].

[18] P. Gondolo and G. Gelmini, Cosmic abundances of stable particles: Improved analysis, Nucl. Phys. B360 (1991) 145. 
[19] F. Karsch, E. Laermann and A. Peikert, The pressure in 2, 2+1 and 3 flavour QCD, Phys. Lett. B478 (2000) 447, [hep-lat/ 0002003 ].

[20] S. Eidelman et al. (Particle Data Group Collaboration), Review of particle physics, Phys. Lett. $\mathbf{B 5 9 2}$ (2004) 1.

[21] M. Battaglia, I. Hinchliffe and D. Tovey, Cold dark matter and the LHC, J. Phys. G30 (2004) R217, [hep-ph/0406147].

[22] K. N. Abazajian and F. G. M., Bulk QCD thermodynamics and sterile neutrino dark matter, Phys. Rev. D66 (2002) 023526, [astro-ph/ 0204293 ].

[23] M. Battaglia et al., Updated post-WMAP benchmarks for supersymmetry, Eur. Phys. J. C33 (2004) 273, [hep-ph/0306219]. 